\title{
First principles of consistent physics
}

\author{
Wanpeng Tant \\ Department of Physics, Institute for Structure and Nuclear Astrophysics (ISNAP), \\ University of Notre Dame, Notre Dame, Indiana 46556, USA
}

(Dated: March 29, 2021)

For a consistent picture of fundamental physics and cosmology, three first principles are proposed as the foundations. That is, quantum variational principle that provides the formalism, consistent observation principle that set physical constraints and symmetries, and spacetime inflation principle that determines physical contents (particle fields and interactions). Under these three principles, a series of supersymmetric mirror models are constructed to study various phases of the universe at different spacetime dimensions and the dynamics between the phases. In particular, mirror symmetry, as the orientation symmetry of the underlying geometry, plays a critical role in the new framework.

Keywords: first principles; mirror symmetry; holonomy symmetry; supersymmetry; fiber bundles; spacetime; renormalization group; phase transitions; inflation

\section{INTRODUCTION}

The approach of first principles has widely been pursued and playing a critical role in the development and history of modern science and mathematics. For example, natural selection is one of the best known principles in biology. In mathematics, axioms play the role of first principles such as the parallel postulate for Euclidean geometry. In physics, first principles have been the ultimate goal for any fundamental theories. Newton's three laws are the foundations of classical mechanics. Maxwell's equations, or the classical U(1) gauge principle in modern terms [1], are the basis of classical electromagnetism.

As a more general first principle, Einstein proposed that physical laws are invariant under any reference frames. For inertial frames of reference (i.e., the Lorentz invariance), it leads to special relativity (while the constancy of the speed of light is a natural consequence of the concept of spacetime). Considering accelerated reference frames (i.e., the equivalence principle), Einstein obtained his general relativity (GR) - the ultimate splendor of classical physics. Intriguingly, GR and other classical theories can also be derived from the wellknown variational principle.

For the development of quantum theory, physicists had to use a different set of principles such as discreteness and gauge symmetry principles and even new concepts like the probability amplitude for the physical state and the operators for observables. However, there are some striking inheritances passed on from classical physics. The original non-relativistic form of quantum mechanics, i.e., the Schrödinger equation, can be obtained from the quantum variational principle - the path integral formulation developed by Feynman [2], an upgrade from the classical variational principle. Even the modern quantum field theory (QFT) [3] can be described under this quantum version of the variational principle by replacing paths

* wtan@nd.edu 
with field configurations in spacetime in the integration. In addition, QFT obeys the Lorentz symmetry as well. The needed gauge invariance for degrees of freedom in local space (or fiber space in modern mathematical language of fiber bundles [4) is also strikingly similar to the Lorentz invariance for 4-d spacetime, in the sense that both Lorentz and gauge groups are holonomy groups of given manifolds in differential geometry.

As such, we see two types of lasting principles that seem to be good for both quantum and classical physics. One is the holonomy (Lorentz/gauge) type of invariance while the other is the variational principle. It is hence very intriguing to generalize such principles for the foundation of all physics. And we could also ask what other first principles are needed for such a foundation. In the end, it is tempting to use the same unified set of first principles, not necessarily a single model or theory, to consistently understand fundamental physics and cosmology including both classical (spacetime) and quantum phenomena.

Ever since the establishment of the Standard Model (SM) of particle physics in 1970s as the current version of QFT, the idea of pursuing theory of everything has become popular among theoretical physicists as the latest approach of first principles for unifying all particles and interactions including gravity. In particular, various efforts have been made to find such a unification theory (i.e., unification of QFT and GR) with candidates such as string theory, loop quantum gravity, etc.

However, we seem to live in a dynamic world as evidenced by the discovery of an expanding Universe and it is definitely at odds with the static picture of an ultimate unified theory for physics. A more compelling hint would be that, instead of going after a single unification theory, we might be better off to pursue a unified fundamental set of first principles leading to a series of consistent theories for all phenomena. Meanwhile, previous work on quantum gravity might be no waste of effort and could just be used for providing various mathematical aspects or describing different physical phases of the same dynamic reality.

The dynamic picture tells us that the time reversal symmetry has to be broken initially and it has to be the first (broken) symmetry in the universe. Whatever first principles we propose have to be able to naturally break this symmetry first in the very beginning. And there is no reason why the current 4-dimensional spacetime, in particular, its dimensions cannot be dynamic. It is probably more natural to consider that spacetime has evolved in a dimension-by-dimension way.

\section{FIRST PRINCIPLES}

Without further ado, we propose and summarize the three first principles for fundamental physics and cosmology:

1. Quantum variational principle: the quantum version of the variation principle (Feynman's path integral formalism) is applied.

2. Consistent observation principle: a measurable finite physical world is assumed.

3. Spacetime inflation principle: spacetime that defines physical contents emerges via dimensional phase transitions (i.e., first time dimension and then space dimensions become inflated).

Principle-1 (the variational principle) is about the approach of theory construction. Many variants of the variational principle have been developed in the history of physics and math. 
For example, Fermat's principle of least time for optics, and Hamilton's principle or the principle of least action for classical mechanics. The quantum version of the variational principle proposed by Feynman is his well-known path integral formulation. This principle shows how to formulate physical laws in mathematical language without invoking any ad hoc laws a priori. In essence, the variational principle presents why math works in physics.

One of the consequences of Principle-2 is that measurable finiteness of the physical world is probably responsible for its appearance. In other words, observation itself constrains what we can actually observe. For a meaningful observation, at least due to human's limitations, all physical quantities have to be finite. By "measurable" here, we assume that one can do consistent measurements. For consistency of finite measurements, we have to introduce well-defined symmetries, e.g., the holonomy group that preserves the metric of the spacetime geometry (with a special case of Lorentz group in 4-d spacetime). That is to say, symmetries emerge from the requirement of consistent observation. Later on, we'll see how this principle coupled with the other two presents us a variety of emerging symmetry groups and particle fields in a dynamic universe.

The first two principles might provide enough tools to construct a static or single-phase theory for a given physical system. But it does not introduce the physical contents or the (time-asymmetric) dynamics involving phase transitions. The third principle on spacetime evolution is critical for invoking varied sets of particles and interactions at different phases of the dynamic universe. First the time dimension was born. Then one space dimension got exponentially extended or inflated to make the universe a 2-d world. Finally two more space dimensions were inflated resulting in our current 4-d spacetime. As a matter of fact, once the time dimension emerges first, the other inflation processes will follow naturally under no further assumption.

To be concise, Principle- 1 provides the conceptual and math formalism, Principle-2 sets the physical constraints and symmetries, and Principle-3 determines the physical contents (particle fields and interactions). Based on these three first principles, the new mirror theoretical framework [5, 6] can be described by a series of Supersymmetric Mirror Models (SMM1 for 1-d time, SMM2 for 2-d spacetime, and SMM4 for 4-d spacetime) for different spacetime phases and corresponding symmetry breaking models of SMM1b, SMM2b, and SMM4b for the dynamics between these phases. Meanwhile, gravity can be understood as an emergent classical phenomenon of smooth spacetime geometry [7].

\section{A. Quantum Variational Principle}

In the quantum variational principle, the quantum probability amplitude is defined as a coherent sum of contributions from all possible configurations [2],

$$
A=\sum_{\text {configurations }} \exp (i \mathcal{S} / \hbar)
$$

or in the form of modern QFT for a given set of particle fields $\{X\}$,

$$
A=\int d\{X\} \exp (i \mathcal{S}(\{X\}) / \hbar) .
$$

Here the contribution of each configuration of particle fields is determined by an exponential factor of $\exp (i \mathcal{S} / \hbar)$ where $\hbar$ is the Planck constant and $\mathcal{S}$ is the action that includes all physics depending on involved symmetries and particle fields. 
Feynman's path integral formalism is probably his greatest scientific contribution, even compared to his Nobel-prize winning work on quantum electrodynamics. Such an elegant and amazing formalism is definitely worth further elaboration. This quantum version of the variational principle beautifully applies both geometry and algebra to physics in a united way. The geometry of physics and its algebraic structures could be described well in the mathematical language of differential geometry.

Hans Bethe, another Nobel laureate, said, "There are two types of genius. Ordinary geniuses do great things, but they leave you room to believe that you could do the same if only you worked hard enough. Then there are magicians, and you can have no idea how they do it. Feynman was a magician." Indeed, Feynman is certainly one of the most admired physicists in 20th century.

One of the most intriguing features of the variational principle is that every path or configuration contributes equally to the probability amplitude. This democracy principle is for equal opportunities but definitely not for equal results. As a matter of fact, most of the contributions from various configurations cancel out each other in the resulting probability. Only the ones near an extremal action (that is maximally symmetric) are most probable.

The variational principle and associated math of differential geometry also ensures the property of quantum superposition and the linear nature of quantum theory as the differential operator is nilpotent or $d^{2}=0$ [1]. It also states that quantumness, represented by $\hbar$, is more fundamental in Mother Nature. Classical physics is emergent as contributions from most paths cancel out each other and decohere while only the most probable path survives. This leads to the principle of least action in classical physics.

Under the variational principle, the action $\mathcal{S}$ contains the essence of a physical model. How to construct $\mathcal{S}$ has been the main focus of physicists. The requirement of finiteness or renormalizability in quantum field theory is needed. Otherwise, the infinities of nonrenormalizable terms in $\mathcal{S}$ will suppress the configuration due to the exponential phase factor. Asymmetries in $\mathcal{S}$ also cancel out each other resulting in a maximally symmetric action for a given model. What kind of symmetries that $\mathcal{S}$ observes will be set by the observation principle. As for the physical contents or what to be used in constructing $\mathcal{S}$, we have to resort to the spacetime principle.

\section{B. Consistent Observation Principle}

This principle requires two distinct properties of observation: finiteness and consistency. It sets constraints on what types of terms are allowable in the action $\mathcal{S}$ of the variational principle. In modern QFT language, finiteness of measurements means that such terms in $\mathcal{S}$ have to be renormalizable.

Consistency that ensures we can make meaningful measurements introduces symmetries in the world. In particular, it gives the holonomy generalization of the Lorentz-type symmetry in spacetime or the gauge invariance in fiber space. The redundant degrees of freedom in a smooth manifold (either the extended spacetime base manifold or the fiber space) are reflected in its corresponding holonomy group. Such a holonomy group is $O(n)$ (orthogonal group) for an $n$-dimensional Riemannian manifold, $U(n)$ (unitary group) for a Kähler manifold, and $S U(n)$ (special unitary group) for a Calabi-Yau manifold. Consistent observation requires that physical laws or the action $\mathcal{S}$ has to obey such holonomy invariance.

The Lorentz $O(1,3)$ invariance is just a special case of the holonomy group in 4-d spacetime. The gauge groups of the Standard Model $U(1) \times S U(2) \times S U(3)$ are just the corre- 
sponding holonomy groups in the local/fiber space as discussed below.

This principle also invokes two critical discrete symmetries, i.e., mirror symmetry and supersymmetry, and their breakings. The idea of mirror symmetry has long been conjectured (first proposed in Ref. [8, further developed later in Refs. [9 15]) since the discovery of parity violation in weak interactions by Lee and Yang [16]. Combined with the spacetime principle, mirror symmetry is nothing but the orientation symmetry of the manifold, which could be broken globally due to the spacetime topology while preserved locally in the fiber space.

For any positive dimensions, $O(n)=O(1) \times S O(n)$ where the simple discrete $O(1)=$ $Z_{2}$ group (containing two elements of $\{1,-1\}$ ) represents the orientation symmetry of a Riemannian manifold leading to the mirror symmetry we will discuss more about later. When $n \geq 2$, supersymmetry also emerges to keep the action renormalizable.

These renormalizability and holonomy conditions can therefore constrain the physics in the action $\mathcal{S}$ dramatically. It also ensures that our universe is understandable in our own eyes.

\section{Spacetime Inflation Principle}

A well-known verse in "Tao Te Ching" [道德经] by Lao Tzu [老子] says that "Tao produced one; one produced two; two produced three; three produced all things" [道生一，一生二, 二生三, 三生万物]. It amazingly and concisely summarizes the big bang dynamics of the early universe and dimensional phase transitions of spacetime. Here Tao could be understood as quantum chaos with no extended spacetime. One means the one time dimension that was first born in the universe. Two means the two-dimensional spacetime that got inflated during the dimensional phase transition. Probably we should replace three with four here. That is, the last step is the fully inflated four-dimensional spacetime that holds everything we have observed in the current universe.

The inflation of spacetime can be imagined like the growth of a fiber bundle. Spacetime that starts at one-dimension is the base manifold of the fiber bundle. Next, the 1-d tangent fiber space grows exponentially out of the 1-d base manifold. Then the total space of the newly formed 2-d bundle serves as the new base manifold to inflate into a double fiber bundle with four extended dimensions. For the sake of consistency, no more dimensional inflation is possible after the 4-d spacetime is formed.

At each stage, spacetime is the fully extended part of the base manifold. At the last stage, as indicated in string theory [17], six additional dimensions are required in the base manifold for consistency. However, these six dimensions remain uninflated forming the Calabi-Yau space for quarks and strong interactions. We will call these unextended fiber and miniature Calabi-Yau manifolds collectively the local space, which governs quantum physics, i.e., introduces particle fields and gauge interactions while the inflated spacetime describes gravity and other classical phenomena.

For an n-dimensional spacetime geometry (or pseudo-Riemannian manifold of signature $(1, n-1)$, to be exact), the maximum symmetry group for metric preservation (in other words, making measurements consistent) or the holonomy symmetry is $O(1, n)=O(1) \times$ $S O(1, n-1)$. This symmetry group is what generalizes the Lorentz invariance and why one can make meaningful measurements at different spacetime points. Obviously the $O(1)=Z_{2}$ group describes the orientation or mirror symmetry of the spacetime geometry.

The complexification of the local space requires even number for its dimensions resulting 
in a Kähler manifold with compatible complex, symplectic, and Riemannian structures. The corresponding tangent fiber space induced by inflated spacetime is then a Kähler manifold with the holonomy group of $U(n)$. The 6 -d Calabi-Yau base manifold can give rise to a fiber space with its holonomy group of $S U(3)$ for strong interactions of quarks. Spacetime might not be orientable globally but the local space is always orientable providing the mirror symmetry between two sets of particles and interactions.

For a given n-dimensional spacetime geometry, the action $\mathcal{S}$ can also be expressed in terms of the Lagrangian density $\mathcal{L}$,

$$
\mathcal{S}=\int d^{n} x \sqrt{|g|}(\mathcal{L}(\{X\}))
$$

where $g$ is the determinant of the spacetime metric and the set of fields $\{X\}$ is determined by the dimension of spacetime. When $n \geq 2$, spinors and supersymmetry start to emerge and spacetime is naturally extended with a superspace of $(x, \theta, \bar{\theta})$ where $\theta$ and $\bar{\theta}$ are anticommuting Grassmann coordinates. In Eq. (3), the Lagrangian density $\mathcal{L}$ takes the usual form in spacetime after the Grassmann variables are integrated out.

A general scalar function $F(x, \theta, \bar{\theta})$ of superspace can be expanded in a power series of finite number of terms up to $\theta^{2} \bar{\theta}^{2}$ for defining the fields allowed in 4-d spacetime [3]. In 1-d time, $\theta$ and $\bar{\theta}$ do not exist so that the general function $F=\varphi(t)$ is trivial, i.e., only a scalar field $\varphi$ can exist. In 2-d spacetime, $F=\phi(x, t)+\theta \lambda(x, t)+\bar{\theta} \bar{\lambda}(x, t)+\theta \sigma_{\mu} \bar{\theta} A^{\mu}(x, t)$ where more fields like Majorana fermion $\lambda$ and gauge boson $A^{\mu}$ are allowed in addition to a new complex scalar $\phi$. In 4-d spacetime, even more fields and degrees of freedom are allowed. In particular, Dirac fermions like leptons and quarks and bosons of more complicated gauge groups start to appear. These particles are also separated into two ordinary and mirror sectors that are decoupled from each other by the mirror symmetry.

The dimensional phase transitions of spacetime and subsequent electroweak and QCD phase transitions are governed by the spontaneous symmetry breaking Higgs-like mechanism from the real scalar $\varphi$ and other scalars of fermion condensates at various stages. These dynamic processes are probably topological and hence more difficult to quantify.

\section{SUPERSYMMETRIC MIRROR MODELS}

Now we can construct the Supersymmetric Mirror Models (SMM1, SMM2, and SMM4) under the new theoretical framework for varied spacetime dimensions using these first principles. Most of the following materials in this section are taken from previous works [5, 6] although with some improvements.

The simplest 1-d Lagrangian can be written as,

$$
\mathcal{L}_{\mathrm{SMM} 1}=\frac{1}{2} \dot{\varphi}^{2}
$$

for a massless real scalar $\varphi$. In this one-dimensional time manifold, the mirror symmetry is the same as the time reversal symmetry $O(1)=Z_{2}$ which is also the whole holonomy group. The time dimension must emerge first in the geometry so that the universe can have its first symmetry - time reversal symmetry spontaneously broken and become dynamic and causal afterwards. In this 1-d Riemannian geometry, there is no intrinsic curvature, that is, gravity does not exist yet. There is no continuous gauge symmetry and no supersymmetry, 
either. That is, no gauge interactions and no spinor particles exist except for a simple real scalar field. We call this model SMM1, or 1-d Supersymmetric Mirror Model, since it is the simplest 1-d extension to other SMM models in higher dimensions.

When $\varphi$ obtains mass via the Higgs-like mechanism, it will spontaneously break the time reversal symmetry under the symmetry breaking model SMM1b,

$$
\mathcal{L}_{\mathrm{SMM} 1 \mathrm{~b}}=\frac{1}{2} \dot{\varphi}^{2}-V\left(\varphi^{2}\right), \quad V\left(\varphi^{2}\right)=\sum_{k=1}^{\infty} \frac{\lambda_{k}}{k !} \mu^{k+1}\left(\frac{\varphi^{2}}{2}\right)^{k}
$$

where $\lambda_{k}$ are dimensionless couplings, $\mu$ represents the energy scale, and odd potential terms of $\varphi$ are omitted as such asymmetries cancel out in the variational principle. Such a general potential for symmetry breaking is renormalizable and this dynamic symmetry breaking process could be studied under the framework of renormalization group (RG) [18].

When the potential $V\left(\varphi^{2}\right)$ starts forming the shape of a Mexican hat, i.e., the $\varphi^{2}$ or mass term becomes negative while the quartic term or higher terms stay positive, the system will inevitably evolve into one of the two emerging new vacuum configurations breaking its $Z_{2}$ (time reversal) symmetry. The time arrow naturally appears due to the breakdown. The first order of the universe is then established: the time order or causality. Indeed, the time reversal symmetry is the first emergent symmetry and also first broken under this consideration.

At the same time, the scalar becomes massive and leads to the inflation of one space dimension, essentially the so-called anomalous dimension of the scalar in the renormalization group formalism [18. Eventually the scalar decays into the next generation of particles in the newly inflated 2-d spacetime that is then governed by SMM2 with the Lagrangian,

$$
\mathcal{L}_{\mathrm{SMM} 2}=-\frac{1}{4} F_{\mu \nu} F^{\mu \nu}+\frac{i}{2}\left(\lambda_{L}^{\dagger} \bar{\sigma}^{\mu} \partial_{\mu} \lambda_{L}+\lambda_{R}^{\dagger} \sigma^{\mu} \partial_{\mu} \lambda_{R}\right)
$$

where the $U(1)$ gauge tensor $F_{\mu \nu}=\partial_{\mu} A_{\nu}-\partial_{\nu} A_{\mu}$ and the Majorana fermion $\lambda$ has to be neutral and does not couple to the gauge field $A_{\mu}$. Both $\lambda$ and $A_{\mu}$ are massless and have two components or degrees of freedom. They form the simplest $N=1$ abelian gauge supersymmetry multiplet $(1,1 / 2)$ with the on-shell Lagrangian of Eq. (6).

The holonomy group of the 2-d spacetime becomes $O(2)=Z_{2} \times U(1)$, which also induces the fiber space as a $U(1)$ Kähler manifold. Both $U(1)$ gauge bosons and Majorana fermions are born in this phase due to the emergence of supersymmetry. Fermions here have to be of Majorana type instead of Dirac type because antiparticle's degrees of freedom can not be set free in 2-d spacetime. Both of the orientation or mirror symmetries of spacetime and the corresponding fiber space are equivalent of the chiral symmetry in this case. It means that the two sectors of particles defined by the mirror symmetry are just two chiral (left- and right-handed) sectors that are completely decoupled from each other. Gravity first appears in 2-d form which can also be described by a 2-d conformal field theory (CFT). As it turns out, the interior of a black hole is also 2-d in nature governed by SMM2 [7].

At the next stage, the phase transition from 2-d to 4-d, the Majorana fermions condense into two massive scalars $\phi$ and $\phi^{\prime}$ under the $\mathrm{N}=1$ pseudo-supersymmetry model SMM2b (see Ref. 5 for details). The massive scalars spontaneously break the symmetries again leading to the inflation of two additional space dimensions. Like the 1-d case, the renormalizable scalar potential of $\phi$ is,

$$
V\left(\phi^{2}\right)=\mu^{2} \sum_{k=1}^{\infty} \frac{\lambda_{k}}{k !}\left(\frac{\phi^{2}}{2}\right)^{k}
$$


that governs the dynamics of the inflation and similarly we can get $V^{\prime}\left(\phi^{\prime 2}\right)$ for $\phi^{\prime}$.

In the end, the two scalars decay into the new generation of particles in 4-d spacetime under SMM4 (see Ref. [5] for details). The new holonomy group, i.e., the Lorentz group $O(1,3)=Z_{2} \times Z_{2}^{\prime} \times S O^{+}(1,3)$ contains two $Z_{2}$ groups for the orientation and time reversal symmetries of spacetime. The quantum counterpart in the fiber space contains also two copies of $Z_{2}$ groups for the mirror or local orientation symmetry and the time reversal or equivalently $C P$ symmetry, respectively. Accompanied with the restricted $S O^{+}(1,3)$ group, one sees the emergence of $U(1)$ and $S U(2)$ gauge groups and three generations of leptons as Dirac fermions (antiparticles now possess separate degrees of freedom) corresponding to the induced tangent fiber space. The particles are separated into two ordinary and mirror sectors for two orientations, respectively.

The 4-d tangent space can have only one copy of $S U(2)$ gauge group that has to be split into $S U_{L}(2)$ for the ordinary sector of particles and $S U_{R}(2)$ for the mirror sector. On the other hand, its maximal torus subgroup, i.e., the Clifford torus $U(1) \times U(1)^{\prime}$ has the two circles embedded independently in their own 2-d part of the 4-d fiber space, so that the full $U(1)$ interactions of both left- and right-handed particles are preserved in the two ordinary and mirror sectors. The three generations of the particles correspond to three independent ways of representing gauge groups of $U(1) \times U(1)^{\prime}$ and $S U(2)$ out of the 4-d fiber space, or three different ways of complexifying the fiber space. Because of the $\mathrm{U}(1)$ independence, charged leptons can not be mixed and can not be degenerate even after they acquire masses later. Only neutral neutrinos could be mixed and become degenerate when they get massive.

Supersymmetry in 4-d spacetime, in addition, demands the emergence of an additional uninflated compact space of six dimensions (i.e., Calabi-Yau space). This 6-d Calabi-Yau space results in a separate set of fermions, i.e., quarks and their $S U(3)$ (color) gauge interactions and consequently the balance of degrees of freedom between gauge bosons and matter fermions for supersymmetry. The fact that the 6-d space is not extended as 4-d spacetime also causes quark confinement. Due to the orientation or mirror symmetry, there are also two sets of quarks and $S U(3)$ interactions in the two sectors, respectively. This 6-d CalabiYau space and its corresponding 6-d fiber space collectively provide another holonomy group - the $U(6)$ gauge group of quark flavors.

In this phase, all particles are massless and the two sectors are completely decoupled in terms of gauge interactions $\left(U_{f}(6) \times S U_{c}(3) \times S U_{w}(2) \times U_{Y}(1)\right)$ although both sectors share the same extended 4-d spacetime geometry or gravity that is described by Einstein's general relativity.

At last, staged quark condensation leads to spontaneous symmetry breaking again and provides masses to the particles (SMM4b for the current universe, see Ref. [5] for details). This dynamic process also accounts for both electroweak and QCD phase transitions and is governed by the potentials of six scalar components corresponding to quark condensation of six flavors,

$$
V\left(\phi_{f}^{2}\right)=-\frac{\lambda_{2 f}}{2} \mu_{f}^{2} \phi_{f}^{2}+\frac{\lambda_{4 f}}{8} \phi_{f}^{4}
$$

where $f=1, \ldots, 6$. Unlike the cases of 1 -d and 2 -d, there are only two (mass and quartic) terms in these potentials as higher order terms are not renormalizable under 4-d spacetime.

The $U(6)$ flavor gauge is almost completely broken and left with only some approximate global symmetries on isopin, baryon number and heavy quark flavor numbers. Intriguingly, neutrinos become degenerate and gain tiny masses due to a very small mass splitting between the two sectors. Therefore, the ordinary sector becomes left-handed while the mirror one 


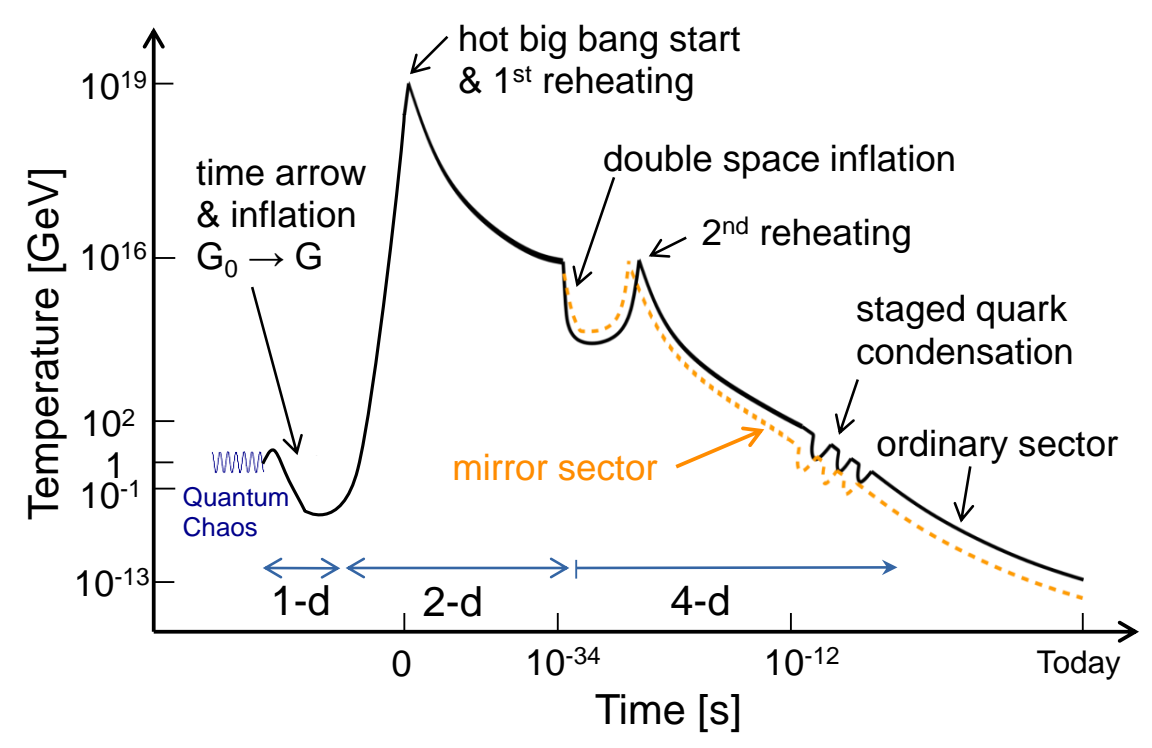

FIG. 1. The schematic diagram (not to scale) is shown for the dynamic history of the universe where the temperature is mostly determined by massless particles and the dips reflect various stages of spontaneous symmetry breaking and emergence of new energy scales leading to each new cosmic era. Adapted from Ref. [5].

right-handed to preserve renormalizability or pseudo-supersymmetry. This gives the wellknown Standard Model of particle physics for the ordinary sector and similar physics for the mirror sector. The two sectors of ordinary and mirror particles are connected via degenerate neutrinos after the symmetry breaking, in particular, the mass terms of neutrinos that involve both left-handed ones in our sector and right-handed ones in the mirror sector.

\section{NEW PICTURE OF THE UNIVERSE}

Applying the Supersymmetric Mirror Models to cosmology, we can see the elegant picture in the evolution of the early universe as depicted in Fig. (1). The time dimension was born first and then the time reversal symmetry got broken leading to the first inflation. The 2- $\mathrm{d}$ spacetime emerged and the hot big bang started at the scale of Planck energy. As the universe cooled down, Majorana fermions started to condense resulting in the process of double space inflation. Eventually 4-d spacetime formed at temperature of $\sim 10^{16} \mathrm{GeV}$ and the two scalar inflatons decayed into massless quarks and leptons in two sectors at different temperatures to reheat the universe. At the scale of $\sim 10^{2} \mathrm{GeV}$, staged quark condensation started a series of phase transitions including electroweak and QCD phase transitions and consequently fermions acquired masses. As the universe has continued to cool down, the ordinary sector has formed stars and galaxies while the mirror sector serves as the dark matter observed today.

However, that is not the full picture of the universe in terms of thermodynamics. Although the universe time flows in one direction, it does not mean that some reversed processes won't occur locally in some corners of the universe. In fact, collapses of stars into black holes go through exactly the reversed process from SMM4b to SMM2. In this sense, these models just describe different phases of spacetime and the dynamics in between as presented in 


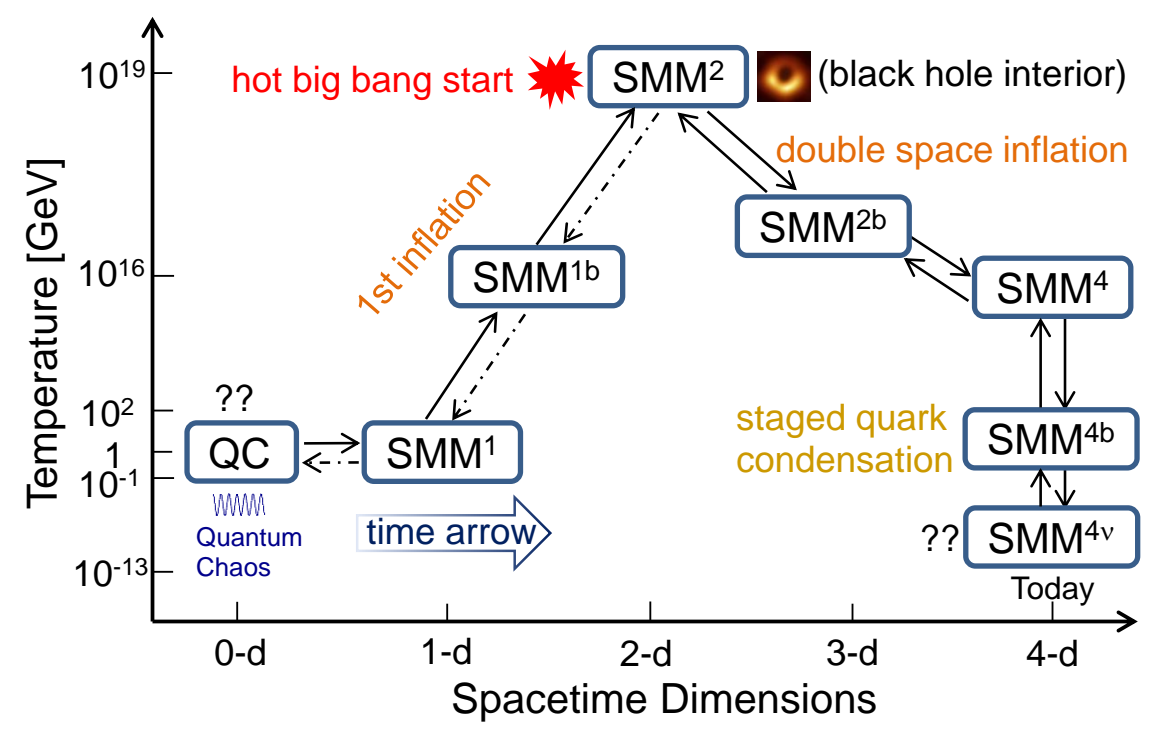

FIG. 2. The schematic diagram (not to scale) is shown for the Supersymmetric Mirror Models at various phases of spacetime. The number in the model name denotes the number of spacetime dimensions while ' $b$ ' indicates that the model corresponds to the dynamic process of spontaneous symmetry breaking between phases. Adapted from Ref. [7].

Fig. (2).

\section{A. Spacetime Phase Transitions and Renormalization Group}

The RG approach has widely been used in studies of critical phenomena and phase transitions [18]. One of the most impressive works using this approach is the discovery of asymptotic freedom of QCD [19, 20, which essentially also tells us why the 6-d Calabi-Yau space is unextended. It seems to be also a good tool to study dimensional phase transitions of spacetime when applying it to the scalar potential theories proposed in Sect. (III).

In 4-d spacetime, the Gaussian fixed point of the coupling $\lambda_{4}$ in the scalar potential is IR-stable, indicating that no more dimensional inflation is possible. In lower dimensions, the Gaussian fixed points of both $\lambda_{2}$ and $\lambda_{4}$ are not stable and tend to make the RG flow to the IR fixed point, leading to dimensional inflation. During each of this RG flow process, the anomalous dimension of the corresponding scalar dynamically changes from zero to one for the growth of one space dimension.

Under the RG picture, SMM1, SMM2, and SMM4 are nothing but description of simple Gaussion fixed points (i.e., with vanishing scalar potentials) of different spacetime configurations. In the early universe, the $\mathrm{RG}$ flow goes from the first Gaussian fixed point (SMM1) to our current phase (end of SMM4b) where we might be at a non-trivial Wilson-Fischer fixed point. Collapse of a massive star into a black hole depicts the reverse process although through a different RG flow trajectory for dimensional collapse of spacetime from 4-d to 2-d. Therefore, black holes are in the simple Gaussian fixed point under 2-d spacetime (SMM2) that can also be described by 2-d conformal field theory (CFT) [7. One may wonder if it is possible for a black hole to continue the collapse into dimensionless quantum chaos, i.e., along the dash-dotted path in Fig. (2). If so, it means that there is an upper limit for a 


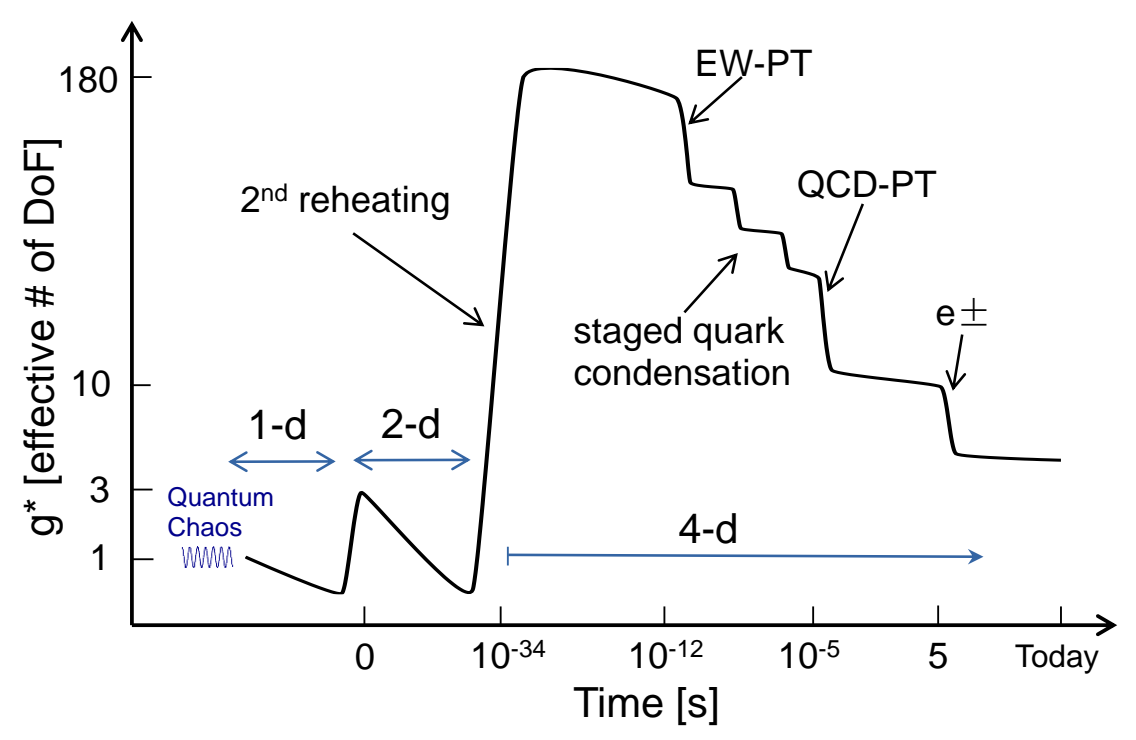

FIG. 3. The schematic diagram (not to scale) is shown for evolution of the effective number of relativistic degrees of freedom $g^{*}$ in the early universe under the Supersymmetric Mirror Models.

black hole's mass.

In the 2-d quantum field theory, the RG method also gives an intriguing result called "c-theorem" 21]. It states that there exists a positive real c-function that decreases monotonically along the RG flow and at the fixed point (2-d CFT), the c-function is the same as the central charge of the Virasoro algebra. Under 2-d CFT, it is shown that the central charge is equal to the effective number of relativistic degrees of freedom $g^{*}$, and in the case of SMM2, we find $c=g^{*}=3$ [7]. Therefore, "c-theorem" can be naturally generalized to " $g$-theorem" for a given spacetime configuration. Due to fermion condensation, symmetry breaking and mass acquiring will effectively reduce the number of degrees of freedom during such a RG flow. Obviously, $g^{*}$ decreases monotonically within each spacetime phase in the early universe as shown in Fig. (3). However, it jumps across the dimensional boundary between two phases. Evidently, $g^{*}$ started out to be one in 1-d time, then peaked as three in 2-d spacetime, and reached its maximum of 180 under 4-d spacetime where contributions from the mirror sector are neglected as it is at lower temperatures.

The generalized " $g$ *-theorem" has interesting consequences. The Gaussian fixed point $\left(\lambda_{k}=0\right)$ in 2 -d spacetime makes $g^{*}$ stationary, i.e., $d g^{*} / d t=0$, with respect to the RG flow. This gives the reason why SMM2 indeed provides UV-stable solutions for black holes. On the other hand, the two ordinary and mirror sectors under 4-d spacetime have different contributions of $g^{*}$ and $g^{*^{\prime}}$ to the degrees of freedom at different temperatures. Such a stationary condition could not typically be met since the collapsing core of a star is mixed evenly with both sectors of matter [22]. Therefore, SMM4 does not give stable configurations in general unless ordinary matter is somehow separated from mirror matter. If such separation indeed occurs, a SMM4 fireball $\left(\sim 10^{16} \mathrm{GeV}\right)$ made of pure ordinary or mirror matter could be UV-stable. 


\section{B. Neutral Particle Oscillations}

Under SMM4b, a small mass splitting of the two ordinary and mirror sectors, on a relative scale of $\delta m / m \sim 10^{-14}$ is predicted. Coupled with the ordinary-mirror quark mixing mechanism, it results in ordinary-mirror neutral hadron oscillations similar to neutrino oscillations between generations [23].

In particular, oscillations of neutrons $\left(n-n^{\prime}\right)$ and kaon mesons $\left(K^{0}-K^{0^{\prime}}\right)$ turn out to be the two most important messengers between the two sectors. These two oscillations present a consistent picture for the origin of both dark matter and baryon asymmetry in the universe [24. The tiny mass splitting scale can also explain the observed scale of dark energy and neutrino masses [25]. In addition, using $n-n^{\prime}$ oscillations we can consistently understand many other puzzles: neutron lifetime anomaly [23], synthesis of heavy elements and stellar evolution [22], ultrahigh energy cosmic rays [26], and unitarity of CKM matrix [27].

Most remarkably, various feasible experiments are proposed to test concrete unique predictions of the oscillation model, including measurement of neutron lifetime anomalies in narrow magnetic traps or under super-strong magnetic fields [27], and detection of unexpectedly large branching fractions of invisible decays of long-lived neutral hadrons [28]. Further evidence of the new physics could be uncovered in the near future.

For example, each incoherent scattering or interaction of a neutral hadron in ordinary matter medium will result in a loss to its mirror counterpart. In terms of neutrons, this loss rate due to $n-n^{\prime}$ oscillations is about $10^{-5}$ per bounce (or $0.45-1 \times 10^{-5}$ as constrained in [28]). This can perfectly explain the so-called neutron lifetime anomaly. It could also be used to further test the new model in neutron lifetime measurements with magnetic traps or super-strong magnetic fields.

\section{Mirror, Super-, and Holonomy Symmetries}

The observation principle invokes three types of critical symmetries. The mirror or orientation symmetry can be considered as the discrete part of the holonomy group. The continuous holonomy groups provide the Lorentz invariance for spacetime itself and gauge groups for interactions. Supersymmetry shows up at 2-d or higher dimensional spacetime for the symmetry between matter fermions and gauge bosons.

Obviously, the local space that defines the quantum properties is always orientable, preserving the mirror symmetry, and hence the quantum world can be divided into two sectors. The orientation symmetry of the spacetime base manifold is part of the Lorentz group and it is the spacetime topology that defines the global orientation or mirror symmetry. If the spacetime geometry is orientable, then the two quantum sectors are completely decoupled. On the other hand, they could become feebly connected if spacetime turns into a nonorientable manifold (like Möbius strip or Klein bottle in 2-d). However, such connections have to be very weak since particles can not change their orientation locally and they have to go around the whole manifold to make the transition. Therefore, quantum transitions between the two sectors are topological in nature.

Spacetime is the stage of all physics and hence the Lorentz invariance inherited from its holonomy is obeyed in both classical and quantum physics. All other continuous gauge symmetries, leading to gauge interactions, come from the holonomy of the local / fiber space (including uninflated space) that is derived from the spacetime base manifold. For a given spacetime geometry, particle fields and gauge interactions are therefore well-defined. 
Supersymmetry is a natural requirement due to finiteness or renormalizability for spacetime with two or more dimensions. It also relates bosons to fermions as the only possible way as indicated by the Coleman-Mandula theorem [29]. It extends the classical spacetime to superspace and introduces both commutative and anti-commutative numbers. It ensures that Hamiltonians or energies are non-negative and the time arrow is preserved in the universe.

However, it is the mirror symmetry instead of supersymmetry that introduces another mirrored copy of particles making up the so-called dark matter of the universe. In our 4-d world, we should search nowhere else for supersymmetry as it's been built even within our own sector, though spontaneously broken, as the symmetry between fermions (leptons and quarks) and gauge bosons (e.g., photons and gluons). The seemingly missing degrees of freedom in bosons can be found in the pseudo-Nambu-Goldstone bosons from the flavor $U(6)$ breakdown 25.

As a metric-preserving symmetry, the holonomy group inevitably and deeply involves the concept of loops (closed paths on a manifold). This is possibly why string theory and loop quantum gravity can be good mathematical or theoretical tools in studying the proposed new framework under certain scenarios. The group equality of $S O(2)=U(1)$ may also indicate why quantum theory in $n \geq 2$ spacetime becomes complex. Furthermore, all these concepts of holonomy, loop, string, and complex are probably profoundly related to each other for $n \geq 2$ spacetime.

\section{Gravity and Spacetime}

Under this new framework, gravity is just the geometry of emergent smooth spacetime as it inflates dimensionally at $n \geq 2$. That is, gravity is truly a classical phenomenon, and also possibly the progenitor of all classical phenomena. Quantum measurement problem is probably related to classical extended spacetime. A measurement can cause a quantum wave function collapsing to its individual eigenstate since the measurement has to be conducted under the metric of the spacetime manifold, which is classical by definition.

Spacetime inflation stops at 4-d and the corresponding gravity theory is just Einstein's general relativity while the simpler 2-d gravity is given in Ref. [7]. Higher dimensional spacetime is not stable or possible. Dimensional inflation requires that only scalar fields exist during the exponential growth. During staged quark condensation including electroweak and QCD phase transitions, other non-condensed fermions (e.g., leptons) exist so that further growth of dimensions is not possible.

The connection between gravity and thermodynamics has been studied since the introduction of the black hole entropy by Bekenstein and Hawking [30, 31. Jacobson derived the Einstein equation of spacetime purely from the consideration of thermodynamics and "downgraded" it to an equation of state [32. Verlinder further developed the idea and treated gravity as an entropic force [33]. As an emergent phenomenon under these studies, it seems to be reasonable to not consider gravity as a fundamental force.

However, as we have shown above, everything is emergent in the scenarios of dimensional evolution of spacetime. Gravity is as essential as other emergent gauge interactions if not more. The coarse-grained description for gravity might be valid mathematically but probably not complete. It has to be combined with the phase transitions in spacetime dimensions. Such a picture of dimensional evolution makes gravity solidify its status in the reality. After each dimensional transition, emergent classical spacetime and gravity are certainly 
TABLE I. as discussed in the text.

\begin{tabular}{c|cc}
\hline \hline Physics & Classical & Quantum \\
\hline fiber bundles & inflated spacetime/base manifold & local/fiber space \\
geometry structures & (pseudo)-Riemannian manifold & Kähler/Calabi-Yau manifolds \\
interactions & gravity & gauge interactions \\
holonomy & $\mathrm{O}(1, \mathrm{n})$ holonomy & $\mathrm{U}(\mathrm{n}) / \mathrm{SU}(\mathrm{n})$ gauge groups \\
mirror symmetry & orientation of spacetime & orientation of local space \\
locality & global & local \\
math & continuum (real) & discreteness (integer) \\
\hline \hline
\end{tabular}

as fundamental as emergent particles and their gauge interactions. Moreover, it is the emergent classical spacetime that determines what kind of quantum physics we would have and it is the inflated spacetime where we live in that selects what quantum physics we could observe.

\section{E. Classic-Quantum Duality}

The details of the classic-quantum duality are listed in Table I. Classical inflated spacetime is emergent from quantum chaos. Classical and quantum properties are two inseparable aspects of the same reality. No observable quantum physics is possible without classical spacetime. Such a duality relationship is probably best depicted in the mathematical language of fiber bundles.

Quantum and classical properties in physics is similar to what discreteness and continuum, or, integer and real numbers in mathematics. In physics, the connections between quantum fiber space and classical spacetime are bridged by the inflation or exponential growth processes. In mathematics, such properties are described by the continuum hypothesis for infinite sets of integers $\left(\aleph_{0}\right)$ and real numbers $\left(\aleph_{1}\right)$, i.e, $\aleph_{1}=e^{\aleph_{0}}$, using an exponentiation operation similar to that in physics. As Cantor introduced, one can easily extend such aleph number series of infinite sets: $\aleph_{0}, \aleph_{1}, \aleph_{2} \ldots$ and even generalize the continuum hypothesis to $\aleph_{\alpha+1}=e^{\aleph_{\alpha}}$ for every ordinal number $\alpha$.

It seems that physics is related to only the first two infinite sets of countable (quantum) $\aleph_{0}$ and uncountable (classical) $\aleph_{1}$. Why don't the higher-ordered infinite sets manifest in our physical world? One possible explanation might be that our spacetime stopped inflating at the critical dimension of four. It is the requirement of consistent measurements that stopped such a possible path to higher-ordered infinities.

\section{CONCLUSIONS AND OUTLOOK}

In summary, Principle-1, the variational principle that provides the math formalism, requires the linear, democratic, and discrete nature of the underlying quantum world under the mathematical language of differential geometry; Principle-2, the observation principle that set physical constraints, leads to the holonomy group (including mirror symmetry) for consistency and renormalizability (including supersymmetry) for finiteness; Principle-3, the spacetime principle that defines physical contents, introduces particle fields and interactions 
of each spacetime phase and the dynamics between the phases. Under these three first principles, the classical spacetime and its quantum contents can then emerge naturally in the universe. In terms of mathematical language of fiber bundles, quantum physics or the local/fiber space is solely determined by the given classical spacetime or the inflated base manifold.

As demonstrated above, all physical phenomena are emergent as the universe unfolds. So are all observed symmetries, all particles, and all laws. And the dynamics consists of a series of dimensional phase transitions in spacetime. Gravity, as a pure classical phenomenon, is about the smoothly inflated spacetime geometry. Quantum theory, on the other hand, describes the non-smooth and discrete part of the local space. The proposed supersymmetric mirror models provide a workable theoretical framework under the first principles.

As Gödel's incomplete theorems have indicated, a consistent math system has to be open and one has to keep on adding more postulates to its existing set of axioms for extending it to unexplored territories. Similarly, these three proposed first principles are, by no means, complete for all physics. We definitely need more guiding principles when we explore various subfields of physics. Even for fundamental physics, there are still profound questions left unanswered.

For example, how does spacetime emerge from dimensionless chaos to 1-d time or what about the details of the emergence of each other dimension? From a distant or classical view, it seems to be fine to use a continuous framework or smooth manifold to describe the evolution of spacetime. But upon a close examination, in particular, around phase transitions, a discrete picture of spacetime may be needed. There are several candidates of theoretical tools that may work for such a picture: loop quantum gravity [34], causal dynamical triangulation [35], causal sets [36], etc.

The critical point is discreteness or quantumness. But how is quantumness realized? There are several descriptive concepts that perhaps tell the same truth or different aspects of the same truth: random, anarchic, democratic, chaotic, self-similar, collective, fractal, universality, cellular automaton, etc. Grasping all these concepts might be essential for understanding the first emergence of time dimension and that of each following individual space dimension. The RG approach shows an interesting way of studying these. But we clearly need better tools to reveal the intriguing details of such dimensional phase transitions in spacetime.

\section{ACKNOWLEDGMENTS}

This work is supported in part by the National Science Foundation under grant No. PHY-2011890. Funding from the faculty research support program at the University of Notre Dame is also acknowledged.

[1] J. Baez and J. P. Muniain, Gauge Fields, Knots and Gravity, Series on Knots and Everything, Vol. 4 (WORLD SCIENTIFIC, 1994).

[2] R. P. Feynman, Rev. Mod. Phys. 20, 367 (1948)

[3] S. Weinberg, The Quantum Theory of Fields (Cambridge University Press, Cambridge, England, 1995). 
[4] B.-Y. Hou and B.-Y. Hou, Differential Geometry for Physicists, Advanced Series on Theoretical Physical Science, Vol. 6 (WORLD SCIENTIFIC, 1997).

[5] W. Tan, Hierarchy of Supersymmetric Mirror Models and Dimensional Evolution of Spacetime, Preprint: https://osf.io/8qawc (Open Science Framework, 2020).

[6] W. Tan, No single unification theory of everything (2020), arXiv:2003.04687 [physics],

[7] W. Tan, From Neutron and Quark Stars to Black Holes, Preprint: https://osf.io/2jywx (Open Science Framework, 2020).

[8] I. Y. Kobzarev, L. B. Okun, and I. Y. Pomeranchuk, Sov J Nucl Phys 3, 837 (1966).

[9] S. I. Blinnikov and M. Y. Khlopov, Sov. Astron. 27, 371 (1983).

[10] E. W. Kolb, D. Seckel, and M. S. Turner, Nature 314, 415 (1985).

[11] H. M. Hodges, Phys. Rev. D 47, 456 (1993).

[12] Z. Berezhiani, Int. J. Mod. Phys. A 19, 3775 (2004).

[13] Z. Berezhiani and L. Bento, Phys. Rev. Lett. 96, 081801 (2006).

[14] J.-W. Cui, H.-J. He, L.-C. Lü, and F.-R. Yin, Phys. Rev. D 85, 096003 (2012).

[15] R. Foot, Int. J. Mod. Phys. A 29, 1430013 (2014).

[16] T. D. Lee and C. N. Yang, Phys. Rev. 104, 254 (1956).

[17] J. Polchinski, String Theory: Volume 2, Superstring Theory and Beyond (Cambridge University Press, 1998).

[18] K. G. Wilson and J. Kogut, Phys. Rep. 12, 75 (1974).

[19] D. J. Gross and F. Wilczek, Phys. Rev. Lett. 30, 1343 (1973).

[20] H. D. Politzer, Phys. Rev. Lett. 30, 1346 (1973)

[21] A. B. Zamolodchikov, JETP Lett. 43, 730 (1986).

[22] W. Tan, Neutron-mirror neutron oscillations in stars (2019), arXiv:1902.03685 [astro-ph, physics:hep-th, physics:nucl-ex].

[23] W. Tan, Phys. Lett. B 797, 134921 (2019), arXiv:1902.01837.

[24] W. Tan, Phys. Rev. D 100, 063537 (2019), arXiv:1904.03835.

[25] W. Tan, Dark energy and spontaneous mirror symmetry breaking (2019), arXiv:1908.11838 [gr-qc, physics:hep-ph, physics:hep-th].

[26] W. Tan, Neutron-mirror neutron oscillations for solving the puzzles of ultrahigh-energy cosmic rays (2019), arXiv:1903.07474 [astro-ph, physics:hep-ph].

[27] W. Tan, Laboratory tests of the ordinary-mirror particle oscillations and the extended CKM matrix (2019), arXiv:1906.10262 [hep-ex, physics:hep-ph, physics:nucl-ex].

[28] W. Tan, Invisible decays of neutral hadrons (2020), arXiv:2006.10746 [hep-ph].

[29] S. Coleman and J. Mandula, Phys. Rev. 159, 1251 (1967).

[30] J. D. Bekenstein, Phys. Rev. D 7, 2333 (1973).

[31] S. W. Hawking, Commun. Math. Phys. 43, 199 (1975).

[32] T. Jacobson, Phys. Rev. Lett. 75, 1260 (1995).

[33] E. P. Verlinde, J. High Energy Phys. 2011, 29, arXiv:1001.0785.

[34] C. Rovelli, Living Rev. Relativ. 11, 5 (2008).

[35] R. Loll, Class. Quantum Grav. 37, 013002 (2019)

[36] S. Surya, Living Rev. Relativ. 22, 5 (2019). 